\title{
SLICING CONVEX BODIES-BOUNDS FOR SLICE AREA IN TERMS OF THE BODY'S COVARIANCE
}

\author{
DOUGLAS HENSLEY ${ }^{1}$
}

\begin{abstract}
Let $Q$ be a zero-symmetric convex set in $\mathbf{R}^{N}$ with volume 1 and covariance matrix $V^{2} \mathrm{Id}_{N \times N}$. Let $P$ be a $K$-dimensional vector subspace of $\mathbf{R}^{n}, K$ $<N$, and let $J=N-K$. Then there exist constants $C_{1}(J)$ and $C_{2}(J)$ such that

$$
V^{-J} C_{1}(J)<\operatorname{vol}_{K}(P \cap Q)<V^{-J} C_{2}(J) \text {. }
$$
\end{abstract}

The lower bound has applications to Diophantine equations.

1. Introduction. The restriction to bodies of covariance a constant multiple of $\mathrm{Id}_{N \times N}$ and volume 1 made in the abstract is not essential, as any centro-symmetric convex body can be brought to that form by a suitable linear transformation. Yet such bodies comprise the most important special cases. The unit cube, the $L^{1}$ ball $\Sigma_{1}^{N}\left|x_{i}\right| \leqslant r$ of volume 1 and the "complex cube" $\left|z_{i}\right| \leqslant \pi^{-1 / 2}, 1 \leqslant i \leqslant N$, are examples.

For the case of the cube, real or complex, only the upper bound is of interest as there is a sharp lower bound of 1 , independent of $K$ and $N$, due to Vaaler [10]. ${ }^{2}$ For the real cube in the case $K=N-1$ an upper bound of 5 was given in [6], which we improve here to $\sqrt{6}$.

Examples with a cube show that $C_{1}(J) \ngtr 12^{-J / 2}$ and $C_{2}(J) \nless 6^{-J / 2}$. We take

$$
\begin{aligned}
& C_{1}(J)=(J+2)^{-J / 2} \bar{\pi}^{J / 2} \Gamma\left(\frac{1}{2} J+1\right), \\
& C_{2}(J)=2\left(8(\log 2)^{-J-3}(J+2) !\right)^{J / 2} \bar{\pi}^{J / 2} \Gamma\left(\frac{1}{2} J+1\right) \text { for } J \geqslant 2,
\end{aligned}
$$

and $C_{2}(1)=1 / \sqrt{2}$. Then we have

THEOREM 1. Let $Q$ be a centro-symmetric convex body in $\mathbf{R}^{N}$ with volume $U$ and diagonal covariance matrix $\left(V_{i}^{2} \delta_{i j}\right), 1 \leqslant i, j \leqslant N$. Let $P$ be a $K$-dimensional vector subspace of $\mathbf{R}^{N}$ with $K<N, K+J=N$. Let

$$
V=\left(U^{-N-2} \prod_{1}^{N} V_{i}^{2}\right)^{1 / 2 N} \text { and } c_{i}=V_{i} V^{-1} U^{-1 / 2}
$$

Let $W_{K}$ be the product of the $K$ smallest $c_{i}$ and $W_{K}^{\prime}$ the product of the $K$ largest $c_{i}$.

Received by the editors January 26, 1979 and, in revised form, October 12, 1979; presented to the Society, August 22, 1979.

AMS (MOS) subject classifications (1970). Primary 52A40, 60D05; Secondary 10B45, 10E05, 52A20. Key words and phrases. Log concave, convex body, covariance, slice area bounds.

${ }^{1}$ This research was supported by National Science Foundation grant \# MCS-7905409.

${ }^{2}$ The lower bound 1 for the case $K=N-1$ is implicit in H. Hadwiger's Gitterperiodische Punktmengen and Isoperimetrie, Monatsh. Math. 76 (1972), 410-418. 
Then

$$
C_{1}(J) W_{K} V^{-J} \leqslant \operatorname{vol}_{K}(P \cap Q)<C_{2}(J) W_{K}^{\prime} V^{-J}
$$

If all the $V_{i}$ are equal to some $V$, and $U=1$ then all $c_{i}, W_{K}$, and $W_{K}^{\prime}$ are 1 and Theorem 1 reduces to

THEOREM 1'. Let $Q$ be a centro-symmetric convex body in $\mathbf{R}^{N}$ with volume 1 and covariance matrix $V^{2} \mathrm{Id}_{N \times N}$. Let $P$ be a $K$-dimensional vector subspace of $\mathbf{R}^{N}, K<$ $N$ and $J=N-K$. Then

$$
V^{-J} C_{1}(J)<\operatorname{vol}_{K}(P \cap Q)<V^{-J} C_{2}(J) .
$$

Theorem 1 follows from $1^{\prime}$ by an elementary lemma whose proof we omit. (But see Example 12, [8].)

LemMa 1. Let $E$ be a convex set in $\mathbf{R}^{N}$ of dimension $K<N$. Let $T$ be a linear transformation which maps each unit coordinate vector $\bar{e}_{i}, 1<i<N$, in $\mathbf{R}^{N}$ to $c_{i} \bar{e}_{i}$, with $c_{i}>0$. Let $E^{\prime}=T E$. Let $W_{K}$ be the product of the $K$ smallest $c_{i}$ and $W_{K}^{\prime}$ the product of the $K$ largest $c_{i}$. Then

$$
W_{K} \operatorname{vol}_{K} E<\operatorname{vol}_{K} E^{\prime}<W_{K}^{\prime} \operatorname{vol}_{K} E .
$$

One may adapt our lower bound to Vaaler [12] by using a variant of his Lemma 6. Suppose $Q \subseteq \mathbf{R}^{N}$ has volume 1 , is centro-symmetric and has covariance matrix $\operatorname{Cov}(Q)=V^{2} \operatorname{Id}_{N \times N}$. Let $L_{j}(\bar{x}), 1<j<N$, be $N$ real linear forms in $K$ variables, $L_{j}(\bar{x})=\sum_{k=1}^{K} a_{j k} x_{k}$, so that $A=\left(a_{j k}\right)$ is an $N \times K$ matrix, with $N>K, N=K+$ $J$.

LEMMA 6 '. Let $M$ be a positive integer and suppose that

$$
M\left|\operatorname{det} A^{T} A\right|^{1 / 2}<V^{-J} C_{1}(J) .
$$

Then there exist at least $M$ distinct pairs of nonzero lattice points $\pm \bar{v}_{m}, 1<m<M$, such that for each $m$,

$$
\bar{l}_{m}=\left(L_{j}\left(\bar{v}_{m}\right)\right) \in 2 Q
$$

With Lemma $6^{\prime}$ in place of Lemma 6 of [12] and following [12] otherwise, we have a generalization of that paper's main result. Let $\Lambda_{j}(\bar{x})=\sum_{k=1}^{K} a_{j k}^{\prime} x_{k}$ for $1<j<J$ be $J$ real linear forms in $K$ real variables $x_{1}, \ldots, x_{K}$. Let $N=J+K$. Let $Q$ be centro-symmetric and convex with $\operatorname{vol}_{N} Q=1$ and $\operatorname{Cov}(Q)=V^{2} \operatorname{Id}_{N \times N}$. For $\bar{y} \in \mathbf{R}^{K}$ let $l(\bar{y})=\left(y_{1}, y_{2}, \ldots, y_{K}, \Lambda_{1}(\bar{y}), \ldots, \Lambda_{J}(\bar{y})\right) \in \mathbf{R}^{N}$.

ThEOREM 2. Let $M$ be a positive integer and suppose that

$$
M^{2} \prod_{1}^{J}\left(1+\sum_{1}^{K}\left|a_{j k}^{\prime}\right|^{2}\right)<C_{1}(J) V^{-J} .
$$

Then there exist $M$ distinct pairs of nonzero lattice points $\pm \bar{v}_{m}$, $1<m<M$, in $\mathbf{Z}^{K}$ such that $l\left( \pm \bar{v}_{m}\right) \in 2 Q$. 
REMARK. When $Q$ is taken to be the unit cube we get a weaker version of Theorem 1 in [12] with all $\alpha_{k}$ and $\beta_{j}$ there being 1 ; our more general lower bound is not as sharp for the cube as that in [11].

2. Proofs. Lemma $6^{\prime}$ follows from the proof in [11] of Theorem 2 (which appears in [12] as Lemma 6), but we use our Theorem $1^{\prime}$ in place of Theorem 1 of [11].

For the proof of Theorem 1 from Theorem $1^{\prime}$ we take the $c_{i}$ in the statement of Theorem 1 as defining $T$, and let $Q^{\prime}=T^{-1} Q, P^{\prime}=T^{-1} P$. Applying Theorem $1^{\prime}$ to $Q^{\prime}$ and then Lemma 1 to the resulting bounds on $\operatorname{vol}_{K}\left(P \cap Q^{\prime}\right)$ gives Theorem 1 .

It remains to prove Theorem $1^{\prime}$. For the proof we shall need some lemmas about centro-symmetric log concave functions. (A function $f: \mathbf{R}^{J} \rightarrow \mathbf{R}^{+} \cup\{0\}$ is $\log$ concave if $\log f$ is concave.)

Lemma 2. Suppose $f: \mathbf{R}^{J} \rightarrow \mathbf{R}^{+} \cup\{0\}$ satisfies $f(\bar{x})=f(-\bar{x}),\{\bar{x}: f(\bar{x})>t\}$ is convex and open for each $t$, and $\int_{\mathbf{R}^{J}} f(\bar{x}) d^{J}(\bar{x})=1$. Suppose further that for all unit $\overline{\boldsymbol{\theta}} \in \mathbf{R}^{J}$,

$$
\int_{\mathbf{R}^{J}}(\bar{x} \cdot \bar{\theta})^{2} f(\bar{x}) d^{J}(\bar{x})<V^{2}
$$

Then

$$
f(\overline{0}) \geqslant V^{-J}(J+2)^{-J / 2} \bar{\pi}^{J / 2} \Gamma\left(\frac{1}{2} J+1\right)=V^{-J} C_{1}(J) .
$$

Proof. Let $h: \mathbf{R}^{J} \rightarrow \mathbf{R}^{+} \cup\{0\}$ be constant at $V^{-J} C_{1}(J)$ for $\|\bar{x}\|<R=$ $V(J+2)^{1 / 2}$, and 0 for $\|\bar{x}\| \geqslant R$. Then $\int_{\mathbb{R}^{\prime}} h=1$ and $\int_{\mathbf{R}^{\prime}} x_{i}^{2} h=V^{2}$, so $h$ satisfies the hypotheses of Lemma 2 . Thus to prove the lemma it suffices to prove that if $f \neq h, f(\overline{0})>h(\overline{0})$.

We first show that $f$ can be replaced by an $f_{1}$ such that $f_{1}$ is circular symmetric, that is, $f_{1}(\bar{x})=f_{1}(\bar{y})$ if $\|\bar{x}\|=\|\bar{y}\|$, and such that $f_{1}(\overline{0})=f(\overline{0})$ and $f_{1}$ satisfies the hypotheses of the lemma.

For let $E_{t}=\{\bar{x}: f(\bar{x})>t\}$. By hypothesis $E_{t}$ is convex, and if $t<s, E_{s} \subseteq E_{t}$. Thus

$$
\int_{\mathbf{R}^{J}} f(\bar{x})=\int_{t=0}^{f(0)} \int_{E_{t}} 1 d^{J}(\bar{x}) d t
$$

while

$$
\int_{\mathbf{R}^{J}} x_{i}^{2} f(\bar{x})=\int_{t=0}^{f(0)} \int_{E_{t}} x_{i}^{2} d^{J}(\bar{x}) d t .
$$

Let $E_{t}^{\prime}$ be the ball about $\overline{0}$ of the same vol, as $E_{t}$, and let $f_{1}(\bar{x})=\sup \left\{t: \bar{x} \in E_{t}^{\prime}\right\}$. Then

$$
\int_{\mathbf{R}^{J}} f_{1}(\bar{x})=\int_{0}^{f(0)} \int_{E_{t}^{\prime}} 1 d^{J}(\bar{x}) d t=\int_{0}^{f(0)} \int_{E_{t}} 1 d^{J}(\bar{x}) d t=\int_{\mathbf{R}^{J}} f(\bar{x})=1 .
$$

Further,

$$
\int_{\mathbf{R}^{\top}} x_{i}^{2} f_{1}(\bar{x})=J^{-1} \int_{\mathbf{R}^{\prime}}\|\bar{x}\|^{2} f_{1}(\bar{x})=J^{-1} \int_{0}^{f(0)} \int_{E_{i}^{\prime}}\|\bar{x}\|^{2} d^{J}(\bar{x}) d t
$$


Now consider a particular value of $t$. If we show that $\int_{E_{t}^{\prime}}\|\bar{x}\|^{2}<\int_{E_{t}}\|\bar{x}\|^{2}$, the claim about $f_{1}$ is proved. So let INT $=E_{t}^{\prime} \cap E_{t}, \mathrm{EX}=E_{t} \backslash E_{t}^{\prime}$ and $\mathrm{EX}^{\prime}=E_{t}^{\prime} \backslash E_{t}$. Then

$$
\int_{E_{t}}\|\bar{x}\|^{2}=\int_{\mathrm{INT}}\|\bar{x}\|^{2}+\int_{\mathrm{EX}}\|\bar{x}\|^{2} \geqslant \int_{\mathrm{INT}}\|\bar{x}\|^{2}+\int_{\mathrm{EX}^{\prime}}\|\bar{x}\|^{2}=\int_{E_{t}^{\prime}}\|\bar{x}\|^{2},
$$

because $\|\bar{x}\|^{2}$ is everywhere in EX at least as large as anywhere in $\mathrm{EX}^{\prime}$ and $\operatorname{vol}_{J_{-1}} \mathrm{EX}=\operatorname{vol}_{J_{-1}} \mathrm{EX}^{\prime}$. Thus $\int_{\mathbf{R}^{J}}\|\bar{x}\|^{2} f_{1}(\bar{x}) d^{J}(\bar{x}) \leqslant \int_{\mathbf{R}^{J}}\|\bar{x}\|^{2} f(\bar{x}) d^{J}(\bar{x})$, and because of the circular symmetry of $f_{1}, \int_{\mathbf{R}^{N}}(\bar{x} \cdot \bar{\theta})^{2} f_{1}(\bar{x}) d^{J}(\bar{x}) \leqslant V^{2}$ for all unit vectors $\bar{\theta}$.

Now suppose the lemma is false and $h(\overline{0})>f(\overline{0})=f_{1}(\overline{0})$. Since $h$ and $f_{1}$ depend only on $\|\bar{x}\|=r$ we shall by an abuse of language write $h(r), f_{1}(r)$ for $h(\bar{x}), f_{1}(\bar{x})$ when $\|\bar{x}\|=r$. (For $r<R, h(r)=h(0)$, as well.) Now

$$
\begin{aligned}
\int_{\mathbf{R}^{J}} f_{1}(\bar{x}) d^{J}(\bar{x}) & =\int_{0}^{\infty} f_{1}(r) J \sigma_{J} r^{J-1} d r=1=\int_{\mathbf{R}^{J}} h(\bar{x}) d^{J}(\bar{x}) \\
& =V^{-J} C_{1}(J) \cdot J \sigma_{J} \int_{0}^{R} r^{J-1} d r .
\end{aligned}
$$

Let $F_{1}(r)=\int_{0}^{r} f_{1}(u) J \sigma_{J} u^{J-1} d u$ and $H(r)=\int_{0}^{r} h(u) J \sigma_{J} u^{J-1} d u$. Since for $r<$ $R, h(0)=h(r)>f_{1}(r)$ while for $r>R, 0=h(r) \leqslant f_{1}(r)$, we have $H(r)>F_{1}(r)$ for $0<r<R$, and $H(r) \geqslant F_{1}(r)$ in any case. Thus

$$
\int_{0}^{\infty} 2 r F_{1}(r) d r<\int_{0}^{\infty} 2 r H(r) d r
$$

and

$$
\int_{0}^{\infty} r^{2} d F_{1}(r)=\int_{0}^{\infty} r^{2} f_{1}(r) J \sigma_{J} r^{J-1} d r>\int_{0}^{\infty} r^{2} d H(r)=\int_{0}^{R} r^{2} h(0) J \sigma_{J} r^{J-1} d r .
$$

In other words,

$$
\int_{\mathbf{R}^{J}}\|\bar{x}\|^{2} f_{1}(\bar{x}) d^{J}(\bar{x})>\int_{\mathbf{R}^{J}}\|\bar{x}\|^{2} h(\bar{x}) d^{J}(\bar{x}),
$$

a contradicition.

REMARK. Log concave centro-symmetric functions which satisfy the covariance hypothesis satisfy the other hypotheses of Lemma 2, as is proved in the preliminary lemma of [2]. Lemma 2 gives a sharp lower bound since an extremal function, $h$, is found. Our next lemma is not as sharp.

Lemma 3. Suppose $f: \mathbf{R}^{J} \rightarrow \mathbf{R}^{+} \cup\{0\}$ satisfies $f(\bar{x})=f(-\bar{x})$, is log concave, and $\int_{\mathbf{R}^{J}} f(\bar{x}) d^{J}(\bar{x})=1$. Suppose further that for all unit $\bar{\theta} \in \mathbf{R}^{J}$,

$$
\int_{\mathbf{R}^{J}}(\bar{x} \cdot \bar{\theta})^{2} f(\bar{x}) d^{J}(\bar{x}) \geqslant V^{2}
$$

Then $f(\overline{0})<V^{-J} C_{2}(J)$, where $C_{2}(J)$ is the same as in Theorem 1 .

Proof. Let $E_{1}=\left\{\bar{x} \in \mathbf{R}^{J}: f(\bar{x})>\frac{1}{2} f(\overline{0})\right\}$. $E_{1}$ is convex and centro-symmetric. Let $E_{i}=i E_{1} \backslash(i-1) E_{1}$ for $i>1$, so that $\mathbf{R}^{J}$ is the disjoint $\cup_{i=1}^{\infty} E_{i}$. Let $r$ be the minimal radius of $E_{1}$, and let $\bar{\theta}$ be a unit vector in the direction of a point on $\partial E_{1}$ of norm $r$. 
Since $\int_{\mathbf{R}^{\prime}}(\bar{x} \cdot \bar{\theta})^{2} f(\bar{x}) d^{J}(\bar{x}) \geqslant V^{2}$,

$$
V^{2} \leqslant \sum_{i=1}^{\infty} \int_{E_{i}}(\bar{x} \cdot \bar{\theta})^{2} f(\bar{x}) d^{J}(\bar{x})<4 \sum_{i=1}^{\infty} i^{J+2} 2^{-i} r^{2}
$$

because vol $E_{1} \leqslant 2 / f(\overline{0})$ and $\int_{E_{1}}(\bar{x} \cdot \bar{\theta})^{2} f(\bar{x}) d^{J}(\bar{x}) \leqslant(2 / f(\overline{0})) r^{2} f(\overline{0})=2 r^{2}$. Now

$$
4 \sum_{1}^{\infty} i^{J+2} 2^{-i} r^{2} \leqslant 8 r^{2} \int_{0}^{\infty} s^{J+2} 2^{-s} d s=D r^{2}=8 r^{2}(\log 2)^{-J-3}(J+2) !
$$

by the integral comparison test. Thus $r \geqslant D^{-1 / 2} V$. On the other hand $2>$ $f(\overline{0}) \operatorname{vol}_{J}\left(E_{1}\right) \geqslant f(\overline{0}) \sigma_{J} r^{J}$ so

$$
\begin{aligned}
f(\overline{0}) & \leqslant 2 r^{-J} \sigma_{J}^{-1} \leqslant 2 V^{-J} D^{J / 2} \sigma_{J}^{-1} \\
& =V^{-J} \cdot 2\left(8(\log 2)^{-J-3}(J+2) !\right)^{J / 2} \pi^{-J / 2} \Gamma\left(\frac{1}{2} J+1\right)=V^{-J} C_{2}(J) .
\end{aligned}
$$

In case $J=1$ we can find the extremal function and obtain a sharper upper bound.

Lemma 4. Suppose $f: \mathbf{R} \rightarrow \mathbf{R}^{+} \cup\{0\}$ satisfies $f(x)=f(-x)$, is log concave, and $\int_{\mathbf{R}} f(x) d x=1, \int_{\mathbf{R}} x^{2} f(x) d x=V^{2}$. Then $f(0) \geqslant 2^{-1 / 2} V^{-1}$.

Proof. Let $\alpha=f(0)$. Since $\int_{-\infty}^{\infty} e^{-2 \alpha|x|} d x=1, f(x)$ cannot be everywhere $\geqslant$ $\alpha e^{-2 \alpha|x|}$. They are equal at 0 , so either $f(x) \equiv \alpha e^{-2 \alpha|x|}$ or there exists $\beta>0$ such that for $0<x<\beta, f(x)>\alpha e^{-2 \alpha|x|}$ while for $x>\beta, f(x)<\alpha e^{-2 \alpha|x|}$. (Both are log concave.)

Now let $F(x)=\int_{0}^{x} f(t) d t$ and $G(x)=\int_{0}^{x} \alpha e^{-2 \alpha|t|} d$. Then

$$
\begin{aligned}
\int_{-\infty}^{\infty} x^{2}\left(f(x)-\alpha e^{-2 \alpha|x|}\right) d x & =2 \int_{0}^{\infty} x^{2}\left(f(x)-\alpha e^{-2 \alpha x}\right) d x \\
& =4 \int_{0}^{\infty} x(G(x)-F(x)) d x
\end{aligned}
$$

since $x^{2}(F-G)$ declines exponentially to zero at $\pm \infty$. Since $F(0)=G(0)=0$ and $F(\infty)=G(\infty)=\frac{1}{2}$, and since $d(F-G) / d x>0$ only for $0<x<\beta$, $x(G(x)-F(x))$ is always negative and $\int_{-\infty}^{\infty} x^{2}\left(f(x)-\alpha e^{-2 \alpha|x|}\right) d x<0$.

What does all this have to do with $\operatorname{vol}_{K}(P \cap Q)$ ? Let $K_{J}$ denote the unit cube $\left|x_{i}\right| \leqslant \frac{1}{2}, K<i \leqslant N$. Let $B$ denote the null matrix of $P$, so that $B$ has $N$ columns and $J$ rows, and $B \bar{x}=\overline{0}$ if $\bar{x} \in P$. Without loss of generality we may assume $\operatorname{rank}(B)=J$. Let $B_{\varepsilon}=\left[B \mid \varepsilon I d_{J \times J}\right]$, and let $P_{\varepsilon}$ be the null space of $B_{\varepsilon}$. Then

$$
\lim _{\varepsilon \rightarrow 0} \operatorname{vol}_{N}\left(P_{\varepsilon} \cap\left(Q \times K_{J}\right)\right)=\operatorname{vol}_{K}(P \cap Q) \text {, }
$$

and

$$
\operatorname{vol}_{N}\left(P_{\varepsilon} \cap\left(Q \times K_{J}\right)\right)=\operatorname{vol}_{N}\left\{\bar{z}: B_{\varepsilon} \bar{z}=0 \text { and } \bar{z} \in Q \times K_{J}\right\} .
$$

We wish to compare the volume in (8) to its projection onto the first $N$ coordinates, $\operatorname{vol}_{N}\left(\left\{\bar{x} \in Q: B \bar{x} \in \varepsilon K_{J}\right\}\right)$. This ratio is $\varepsilon^{-J}\left(\operatorname{det} B_{e} B_{e}^{T}\right)^{1 / 2}$, which we now prove.

We shall need a lemma about how areas are affected by projection. If $\bar{a}_{1} \ldots \bar{a}_{N}$ are column vectors in $\mathbf{R}^{N+J}$, if $A=\left[\bar{a}_{1} \bar{a}_{2} \ldots \bar{a}_{N}\right]^{T}$ is a matrix of $N+J$ columns 
and $N$ rows, and $\operatorname{Box}_{A}=\left\{\bar{a}: \bar{a}=\Sigma_{1}^{N} \lambda_{i} \bar{a}_{i}, 0<\lambda_{i}<1\right\}$ then $\operatorname{vol}_{N} \operatorname{Box}_{A}=$ $\left(\operatorname{det} A A^{T}\right)^{1 / 2}$ (Eves [3]).

Now suppose $A$ has the form $\left[\operatorname{Id}_{N \times N} \mid-D\right]$ and let $A^{\prime}=\left[D^{T} \mid \operatorname{Id}_{J \times J}\right]$.

Lemma 5. $\operatorname{Det}\left(A A^{T}\right)=\operatorname{Det}\left(A^{\prime} A^{\prime T}\right)$.

Proof. By the Cauchy-Binet theorem, $\operatorname{Det}\left(A A^{T}\right)=\Sigma_{\alpha}(\operatorname{det} \alpha)^{2}$ where the summation is over all $N \times N$ minors $\alpha$ of $A$ [5]. Now a typical $\alpha$ consists of $l$ columns of $\operatorname{Id}_{N \times N}$ and $m$ columns of $D, l<N, m<J, l+m=N$.

$$
\alpha=\left(\bar{e}_{\alpha_{1}} \bar{e}_{\alpha_{2}} \cdots \bar{e}_{\alpha_{1}}-\bar{D}_{\alpha_{1+1}}-\bar{D}_{\alpha_{2+1}}-\cdots-\bar{D}_{\alpha_{m+1}}\right) \text {. }
$$

Expanding det $\alpha$ about the 1st through $l$ th columns, each of which contain 1 once and otherwise zeros, we have $\operatorname{Det}(\alpha)= \pm \operatorname{Det}(\beta)$ where $\beta$ is the square matrix consisting of the intersection of the $N-l$ rows not indexed by $\alpha_{1}, \alpha_{2} \ldots \alpha_{l}$ and the $m$ columns of $-D$ belonging to $\alpha$. Thus $\operatorname{det}\left(A A^{T}\right)=1+\Sigma_{\beta}(\operatorname{det} \beta)^{2}$, the sum taken over all $m \times m$ minors of $D, m<J$. But $\operatorname{Det}\left(A^{\prime} A^{\prime T}\right)=\Sigma_{\gamma}(\operatorname{det} \gamma)^{2}$, sum over $J \times J$ minors $\gamma$ of $A^{\prime}$, and these may be expanded about the columns of $\operatorname{Id}_{J \times J}$ to obtain again $1+\Sigma_{\beta}(\operatorname{det} \beta)^{2}$.

Now let $b_{i}, 1<i<N$, denote the column vectors of $B$. Let

$$
\bar{a}_{i}=\left[\frac{\bar{e}_{i}}{-\varepsilon^{-1} \bar{b}_{i}}\right] \text { and } A^{T}=\left[\bar{a}_{1} \bar{a}_{2} \ldots \bar{a}_{N}\right] \text {. }
$$

Then $B_{e} A^{T}=\left(0_{J \times N}\right)$ so that the $\bar{a}_{i}$ are vectors in $\operatorname{Null}\left(B_{\varepsilon}\right)$. When these $\bar{a}_{i}$ are projected onto their first $N$ coordinates they are $\bar{e}_{1}, \bar{e}_{2} \ldots \bar{e}_{N}$. Therefore they are linearly independent and form a basis of $\operatorname{Null}\left(B_{\varepsilon}\right)=P_{\varepsilon}$.

Now vol $\operatorname{Box}_{A}=\left(\operatorname{det} A A^{T}\right)^{1 / 2}$ while $\operatorname{vol}_{N} \operatorname{Proj}\left(\operatorname{Box}_{A}\right)=1$ as $\bar{e}_{1} \ldots \bar{e}_{N}$ are orthonormal. The ratio of volumes, $\left(\operatorname{det} A A^{T}\right)^{1 / 2}$, is independent of which measurable set is projected.

Now $A$ has the form $A=\left[\operatorname{Id}_{N \times N} \mid-D\right]$ with $D=\varepsilon^{-1} B^{T}$, so that $A^{\prime}=$ $\left[\varepsilon^{-1} B \mid \mathrm{Id}_{J \times J}\right]$. Thus

$$
\begin{aligned}
\left(\operatorname{det} A A^{T}\right)^{1 / 2} & =\left(\operatorname{det} A^{\prime} A^{\prime T}\right)^{1 / 2} \text { by Lemma } 5 \\
& =\varepsilon^{-J}\left(\operatorname{det} B_{\varepsilon} B_{\varepsilon}^{T}\right)^{1 / 2},
\end{aligned}
$$

and this is the ratio of a volume in $P_{e}$ to the volume of its projection onto the first $N$ coordinates, as claimed.

Since we can find $\operatorname{vol}_{N}\left(P_{\varepsilon} \cap\left(Q \times K_{J}\right)\right)$ from $\operatorname{vol}_{N}\left\{\bar{x} \in Q: B \bar{x} \in \varepsilon K_{J}\right\}$, we turn our attention to this last. It may be regarded as the probability that an $\bar{x}$ taken "at random" from $Q$ (the probability measure being Lebesgue measure restricted to $Q$ ) will satisfy $B \bar{x} \in \varepsilon K_{J}$. Let $f(\bar{x})$ denote the probability density function of $B \bar{x}$. Since $Q$ is convex, $f(\bar{x})$ is log concave. (This is the key observation.)

For let $\mu$ denote the probability measure associated with $f$, and $\nu$ Lebesgue measure restricted to $Q$. Let $s=1-t, 0<t<1$, and let $C, D$ be open convex sets in $\mathbf{R}^{J}$. Let $B^{-1} C, B^{-1} D$ be the inverse images in $\mathbf{R}^{N}$ under $B$ of $C$ and $D$ respectively. From Prekópa [9], since $\chi_{Q}$, the characteristic function of $Q$, is $\log$ concave, the measure $\nu$ is $\log$ concave, that is, $\nu\left(s C^{\prime}+t D^{\prime}\right)>\left(\nu\left(C^{\prime}\right)\right)^{s}\left(\nu\left(D^{\prime}\right)\right)^{t}$. Let 


$$
\begin{aligned}
C^{\prime}=B^{-1} D, D^{\prime} & =B^{-1} D . \text { Then } \\
\mu(s C+t D) & =\nu\left(B^{-1}(s C+t D)\right)=\nu\left(s B^{-1} C+t B^{-1} D\right) \quad(\text { since } B \text { is linear) } \\
& =\nu\left(s C^{\prime}+t D^{\prime}\right)>\left(\nu\left(C^{\prime}\right)\right)^{s}\left(\nu\left(D^{\prime}\right)\right)^{t}=(\mu(C))^{s}(\mu(D))^{t} .
\end{aligned}
$$

So $\mu$ is a log concave measure and, again by Prekópa [9], $f$ is a log concave function. Now as $\varepsilon \rightarrow 0$,

$$
\operatorname{Prob}\left(B \bar{x} \in \varepsilon K_{J}\right) \sim \varepsilon^{J} f(\overline{0})
$$

Also,

$$
\operatorname{Cov}(f)_{i j}=\int_{\mathbf{R}^{\prime}} x_{i} x_{j} f(\bar{x}) d^{J}(\bar{x})=\left(V^{2} B B^{T}\right)_{i j}
$$

Let $\bar{X}$ be the random vector uniformly distributed on $Q$. Since $B B^{T}$ is selfadjoint and positive definite (rank $B=J$ ), there is a square matrix $S$ such that $S S^{T}=$ $B B^{T}[1]$.

Let $Y$ be the random vector $\bar{Y}=S^{-1} B \bar{X}$. Then $\operatorname{Cov}(B \bar{X})=V^{2} B B^{T}$, so $\operatorname{Cov}(\bar{Y})$ $=V^{2} S^{-1} B B^{T} S^{-1 T}=V^{2} I d_{J \times J}$, since for any random vector $\bar{Z}$, any square matrix $U, \operatorname{Cov}(U \bar{Z})=E\left(U \bar{Z}(U \bar{Z})^{T}\right)=E\left(U \bar{Z} \bar{Z}^{T} U^{T}\right)=U E\left(\bar{Z} \bar{Z}^{T}\right) U^{T}=U \operatorname{Cov}(\bar{Z}) U^{T}$ because expectation $(E)$ is linear.

Let $h(\bar{x})$ be the probability density function associated with $\bar{Y}$. Then

$$
\begin{aligned}
h(\overline{0}) & =\left(\operatorname{det} B B^{T}\right)^{1 / 2} f(\overline{0}), \\
\operatorname{Cov}(h) & =\operatorname{Cov}(\bar{Y})=V^{2} \operatorname{Id}_{J \times J},
\end{aligned}
$$

and since $\log$ concavity is preserved under the linear transformation $S^{-1}, h$ is $\log$ concave.

Applying Lemmas 2 and 3 to $h$, and Lemma 4 in case $J=1$, we have from (12) that

$$
C_{1}(J) V^{-J}<h(\overline{0})<C_{2}(J) V^{-J}
$$

and from (7) through (11) that

$$
C_{1}(J) V^{-J}<\operatorname{vol}_{K}(P \cap Q)<C_{2}(J) V^{-J} \text {. }
$$

\section{BIBLIOGRAPHY}

1. L. Breiman, Probability, Addison-Wesley, Reading, Mass., 1968, p. 239.

2. Ju. S. Davidovic, B. I. Korenbljum and B. I. Hacet, A property of logarithmically concave functions, Dokl. Akad. Nauk SSSR 85 (1969) = Soviet Math. Dokl. 10 (1969), 477-480.

3. H. Eves, Elementary matrix theory, Allyn and Bacon, Boston, Mass., 1966, p. 176.

4. W. Fleming, Functions of several variables, Springer-Verlag, New York, 1977.

5. F. Gantmacher, Matrizenrechnung, Vol. 1, Deutsche Verlag der Wissenschaften, Berlin, 1970.

6. D. Hensley, Slicing the cube in $\mathbf{R}^{n}$ and probability, Proc. Amer. Math. Soc. 73 (1979), 95-100.

7. M. Kanter, Unimodality and dominance for symmetric random vectors, Trans. Amer. Math. Soc. 229 (1977), 65-85.

8. M. Marcus and H. Minc, Introduction to linear algebra, Macmillan, New York, 1965, p. 209.

9. A. Prekópa, On logarithmic concave measures and functions, Acta Sci. Math. (Szeged) 34 (1973), 335-343.

10. Y. Rinnot, On convexity of measures, Ann. Probability 4 (1976), 1020-1026.

11. J. Vaaler, A geometric inequality with applications to geometry of numbers, Pacific J. Math. (to appear).

12. On linear forms and Diophantine approximation, Pacific J. Math. (to appear).

Department of Mathematics, Texas A\&M Universtry, College Station, Texas 77843 